\title{
The Prognostic Value of CD44 Expression in Epithelial-Mesenchymal Transition: Preliminary Data from Patients with Gastric and Esophageal Cancer
}

\author{
DIMITRIOS SCHIZAS ${ }^{1,2}$, DEMETRIOS MORIS ${ }^{3}$, PRODROMOS KANAVIDIS ${ }^{1}$, ADAMANTIOS MICHALINOS ${ }^{2}$, \\ ATHANASIOS SIOULAS ${ }^{4}$, KITTY PAVLAKIS ${ }^{5}$, ANASTASIOS MACHAIRAS ${ }^{1}$ and THEODOROS LIAKAKOS ${ }^{1,2}$ \\ ${ }^{I}$ Third Department of Surgery, Attikon General Hospital, Medical School, \\ National and Kapodistrian University of Athens, Athens, Greece; \\ ${ }^{2}$ First Department of Surgery, Laikon General Hospital, Medical School, \\ National and Kapodistrian University of Athens, Athens, Greece; \\ ${ }^{3}$ Department of Immunology, Lerner Research Institute, Cleveland Clinic, Cleveland, OH, U.S.A.; \\ ${ }^{4}$ Department of Interdisciplinary Endoscopy, University Medical Center Hamburg-Eppendorf, Hamburg, Germany; \\ ${ }^{5}$ Department of Pathology, Medical School, National and Kapodistrian University of Athens, Athens, Greece
}

\begin{abstract}
Background: Epithelial-mesenchymal transition is one of the mechanisms that contribute to the aggressiveness of cancer. CD44 antigen is an emerging biomarker that is currently being evaluated in the literature in the frame of epithelial-mesenchymal transition. The aim of this study was to evaluate the expression of CD44 in relation to several clinicopathological parameters in gastric and esophageal carcinomas. Materials and Methods: This historical cohort survey was performed on gastric and esophageal tumors obtained from 86 patients who underwent resection from 2003 until 2011. Immunohistochemistry was used for assessing the expression of CD44 with a semiquantitative model. Results: Survival rates were negatively correlated with $p T$ at diagnosis $(p<0.01), p N(p<0.01)$, positive lymph node ratio $(p<0.01), p M(p=0.004)$, tumor stage $(p<0.01)$ and grade $(p<0.01)$, and the absence of $R 0$ resection $(p<0.01)$. Disease-free survival rates were affected by $p N$ at diagnosis $(p=0.02)$, positive lymph node ratio $(p=0.03)$ and tumor stage $(p=0.02)$. The correlation of tissue CD44 expression with overall and disease-free survival was not statistically significant, whereas tumor $T, N$, positive lymph node ratio and lymphovascular invasion were significantly associated with CD44 positivity. Moreover,
\end{abstract}

Correspondence to: Demetrios Moris, MD, M.Sc., Ph.D., MACS, Lerner Research Institute, Cleveland Clinic, 9500 Euclid Ave., NE60, Cleveland, Ohio 44195, U.S.A. Tel: +1 2164442574, Fax: +1 2164454658, e-mail: dimmoris@yahoo.com

Key Words: Gastric adenocarcinoma, esophageal carcinoma, CD44, epithelial-mesenchymal transition, survival, immunochemistry. tissue CD44 expression was not significantly associated with stage, histological subtype according to Lauren classification, the patient's age or gender. Conclusion: CD44 antigen expression might be an indicative and descriptive marker of epithelial-mesenchymal transition, mainly in gastric cancer, reflecting diverse clinicopathological events.

Epithelial-mesenchymal transition (EMT) is a welldescribed embryological process that is considered to play a vital role in tumor progression (1-4). During this process, epithelial cells undergo a phenotypic switch to mesenchymal cells that are similar in appearance to fibroblasts $(1,2)$. The change in cell type results in the loss of polarity and also the loss of tight intracellular adhesions maintained by epithelial cells via adherens junctions $(1,2)$. The aberrant induction of EMT in vitro in adult epithelia led to increased metastatic potential of the cells $(5,6)$.

Increasing evidence suggests that cancer stem cells within malignant tumors reflect the potential for the initiation and progression of cancer, by inducing heterogeneity, metastasis and therapeutic resistance, thus resulting in the poor prognosis of patients $(7,8)$. Furthermore, special biomarkers represent the specific properties of cancer stem cells (9-11). Amongst the several stem cell surface markers, the class I transmembrane glycoprotein CD44 family represents a novel and most robust surface marker especially for stem cells in gastric cancer (GC) (9-11). Studies have suggested CD44 to be an important stem cell marker for multiple solid tumors $(9,10,12)$ and CD44 family proteins can also mediate EMT (13-15).

Therefore, we decided to investigate the immunohistochemical expression of CD44 as a representative marker of 
EMT in patients with gastric and esophageal cancer (EC) and to assess its possible predictive relevance in future clinical practice.

\section{Materials and Methods}

Participants. This historical cohort survey was performed on GC and EC obtained from 86 (61 men and 25 women) patients, aged from 27 to 96 years, who underwent radical resection at the Third Department of General Surgery, Attikon General Hospital, National and Kapodistrian University of Athens, Athens, Greece in 20032011. Informed consent was obtained from all patients. The study was approved by the Ethics Committee of Attikon General Hospital, National and Kapodistrian University of Athens, Athens, Greece, (Ref. No 3/5-3-2012). No patient received any neoadjuvant therapy (neither chemotherapy nor radiotherapy). Information about age and gender were obtained from patients records. Institutional Review Board approval was obtained through the same permission.

One representative tissue block from each case was used for immunohistochemical evaluation. Following the pathology report, histopathological features of the tumor comprising depth of tumor invasion, grade, histological subtype according to Lauren classification, lymphovascular space invasion and regional lymph node involvement were noted. TNM staging was performed according to the seventh edition of the American Joint Committee on Cancer Staging Manual.16 16Patients were followed-up to June 1 st 2015. The clinical endpoints of the study were overall survival and disease-free survival (DFS).

Immunohistochemistry. Staining was performed using the standard streptavidin-biotin-peroxidase complex method with an automated staining system (Autostainer Plus; Dako, Glostrup, Denmark). The tissue sections on the slides were de-paraffinized and rehydrated. For antigen retrieval, the slides were heated in a microwave oven for $15 \mathrm{~min}$ in $10 \mathrm{mM}$ citrate buffer $(\mathrm{pH} \mathrm{6.0)}$. Endogenous peroxidase was blocked with $3 \%$ hydrogen peroxide for $20 \mathrm{~min}$. The slides were then incubated with diluted primary antibody for $30 \mathrm{~min}$ at room temperature. The antibody used was anti-CD44 (H-CAM) (mouse monoclonal, 1:200; Novocastra, Newcastle, UK). After washing with Tris-buffered saline, tissue sections were incubated with biotinylated secondary antibody and then with diaminobenzidine substrate provided in a Dako Envision kit (Dako). Slides were counterstained with Harris hematoxylin. Appropriate positive controls, and negative controls by omitting the primary antibody, were included with each slide run.

Evaluation of samples. Protein expression was assessed using the widely accepted HSCORE system (16). Only membranous CD44 staining was considered as positive. The evaluation of immunohistochemistry was performed in a blinded fashion by a single expert observer (KP). The proportion of neoplastic cells featuring a membranous staining throughout the tumor section was assessed using a low-power magnification $(\times 40)$. The HSCORE was calculated using the following equation: HSCORE $=\sum P i(\mathrm{I})$, where I represents the staining intensity score (i.e. $0=$ no staining, $1=$ weak staining, $2=$ moderate staining, and $3=$ strong staining) and $\mathrm{Pi}$ represents the percentage of stained cells (from 0 to 100\%). The final HSCORE ranged from 0 to 300 and CD44 expression levels were classified as negative and positive using a cut-off value of 30 (17).
Statistical analysis. Statistical analysis was carried out using R language and environment for statistical computing (http://www.Rproject.org). In order to describe data, we used the mean \pm standard deviation, median (range), or frequency, whereas $\chi^{2}$ test, Fisher's exact test, Student's or Welch's $t$-test, and Wilcoxon rank-sum test were used to compare results between groups when appropriate. The Kaplan-Meier method was used to estimate the overall and diseasefree survival rate, and survival differences were analyzed using logrank tests. $p$-Values of less than 0.05 were considered statistically significant.

\section{Results}

This study included 58 patients with GC, 25 patients with gastroesophageal junction cancer and three patients with EC, with a mean age of $68.5 \pm 13.2($ range $=27-96)$ years and a male/female ratio of $2.44: 1$.

The median follow-up period was 59.1 months with a total of 80418 person-days included in the study. At the end of the follow-up, seven patients had been lost to follow-up $(8.1 \%)$, 23 patients $(26.7 \%)$ were alive, and 56 dead, giving a disease-specific mortality of $65.1 \%$. The median censorship interval was 26.7 months.

As far as the location of the tumors is concerned, 32 lesions were found at the body of the stomach, 26 were found at the antrum, 25 were found at the gastroesophageal junction (any Siewert classification) and three in the esophagus. Consequently, two patients underwent central gastrectomy, 43 subtotal gastrectomy, 21 total gastrectomy, 18 esophagogastrectomy and two patients underwent total esophagogastrectomy.

Histologically, 81 malignancies were adenocarcinomas (94.2\%), three were adenosquamous and two squamous cell carcinomas. Tumor TNM, grade, stage, resection status, histological subtype according to Lauren classification and extent of lymph node dissection are shown in Table I.

Survival rates were negatively correlated with pT at diagnosis $(p<0.01), \mathrm{pN}(p<0.01)$, positive lymph node ratio $(p<0.01), \mathrm{pM}(p=0.004)$, stage $(p<0.01)$, grade $(p<0.01)$ and the absence of R0 resection $(p<0.01)$. Disease-free survival rates were affected by $\mathrm{pN}$ at diagnosis $(p=0.02)$, positive lymph node ratio $(p=0.03)$ and stage $(p=0.02)$. Finally we noted a marginal but insignificant tendency for correlation between Lauren diffuse histological type and decreased survival $(p=0.08$, odds ratio $=1.6)$.

Immunohistochemistry data analysis. This study analyzed 86 carcinomas. Of those, 65 cases $(78.3 \%)$ showed positive CD44 expression by immunohistochemistry. The correlation of immunohistochemical CD44 expression with overall and disease-free survival was not statistically significant, whereas tumor $\mathrm{T}$ - and $\mathrm{N}$-stage, positive lymph node ratio and lymphovascular invasion were significantly associated with positive CD44 status (Table II). Moreover, tissue CD44 


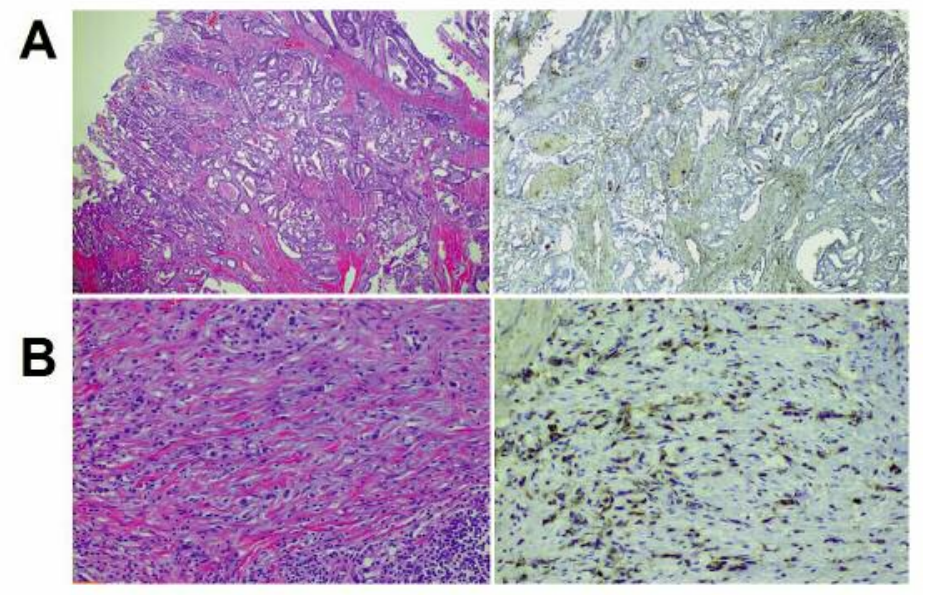

Figure 1. Representative images of immunohistochemical expression of CD44. A: Well-differentiated intestinal-type carcinoma stained by hematoxylin and eosin ( $H$ and $E$ ) stain (left) and for CD44 (right) showing absence of CD44 expression. B: Poorly differentiated diffuse-type adenocarcinoma stained by $H$ and $E$ (left) with positive CD44 expression (right). Original magnification $\times 100$.

expression was not significantly associated with stage and histological subtype according to Lauren classification nor with the patient's age or gender. Nevertheless, a marginal but insignificant tendency for correlation between Lauren diffuse histological type and increased CD44 expression ( $p=0.07$, Odds Ratio=1.6) was noted (Figure 1).

\section{Discussion}

The role of CD44 in the neoplastic process has been explored for nearly 30 years, with numerous studies focusing on the diagnostic and prognostic significance of CD44 expression in human tumors, especially GC. In 1982, CD44 was identified as a surface glycoprotein and a lymphocytehoming receptor found on lymphoid and epithelial cells (18) its main function on lymphocytes is mediating interaction with the endothelium, but initially its function on epithelial cells was not entirely understood (19).

Studies have revealed that one of the major functions of CD44 is in epithelial-mesenchymal transition (20-22) its overexpression being indicative of poor prognosis and recurrence (22) in a variety of tumor types (23-26). Specifically, CD44 is an adhesion/homing molecule with a pre-oncogenic function since it plays a central role in the remodeling and degradation of hyaluronic acid, a major component of the extracellular matrix. These functions enable cell migration and promote cancer invasion and metastasis. Moreover, the cytoplasmic tail of CD44 recruits ezrin-radixin-moesin proteins that are linked to the actin cytoskeleton and thereby promote cell motility. Interaction between moesin and CD44 is known to be an important process in the EMT of tumor cells $(20,27)$. Nevertheless, Jung et al. demonstrated that the expression of CD44 was an independent indicator of poor prognosis but was not correlated with other clinicopathological factors, nor with moesin expression (28).

This discrepancy may be due to the multiple roles of CD44 in cancer progression, unrelated to moesin expression, since CD44 interacts with diverse proteins and matrix molecules by genetic and epigenetic mechanisms to maintain stem cell features of tumor cells (23).

In the current study, we demonstrated that more than $70 \%$ of the patients with malignancy had moderate to high expression of CD44. In fact, this finding is striking and one of the highest reported in the literature. Jung et al. also demonstrated in their series that $34.0 \%$ of patients with GC had moderate to high expression of CD44 (28) with the current literature varying from $27-62 \%(10,29)$.

Of interest though, the cases of our study featuring high expression of CD44 were not correlated with decreased overall survival. This finding is contrary to that of Jung et al. who demonstrated significant differences only in highly expressing CD44 patients and overall survival using KaplanMeier curves and independently of pathological TNM stage and histological grade (28).

In the same frame, another retrospective study of patients with GC evaluated the expression of CD44 and its prognostic importance, concluding that this cell adhesion molecule is highly expressed in GC. Moreover, expression of CD44 correlated with a poor prognosis in patients with the intestinal type of GC (25). Our findings did not confirm this result, since we found a marginal but not significant tendency for positive correlation between CD44 expression and diffuse-type GC.

Similarly, Yong et al. demonstrated that CD44 was not a reliable marker in identifying the risk of recurrence in patients with GC (17). 
Table I. Clinicopathological data of patients and log-rank p-value for correlation with survival and disease-free survival.

\begin{tabular}{|c|c|c|c|c|}
\hline \multirow[b]{2}{*}{ Characteristic } & \multicolumn{4}{|c|}{ Log-rank $p$-value } \\
\hline & Mean age $( \pm \mathrm{SD})$ years & Patients, n (\%) & Overall survival & Disease-free survival \\
\hline Gender & & & 0.804 & 0.409 \\
\hline Male & $68.6(11.3)$ & $61(70.9 \%)$ & & \\
\hline Female & $68.0(17.1)$ & $25(29.1 \%)$ & & \\
\hline Overall & $68.5(13.2)$ & $86(100 \%)$ & & \\
\hline Age & & & 0.32 & 0.79 \\
\hline$>70$ & & $43(50 \%)$ & & \\
\hline$<70$ & & $43(50 \%)$ & & \\
\hline Histological tumor location & & & 0.15 & 0.53 \\
\hline Stomach & & $58(67.4 \%)$ & & \\
\hline Gastroesophageal junction & & $25(29.1 \%)$ & & \\
\hline Esophagus & & $3(3.4 \%)$ & & \\
\hline $\mathrm{pT}^{*}$ & & & $<0.01$ & 0.09 \\
\hline Tis & & $1(1.2 \%)$ & & \\
\hline $\mathrm{T} 1$ & & $13(15.3 \%)$ & & \\
\hline $\mathrm{T} 2$ & & $11(12.9 \%)$ & & \\
\hline $\mathrm{T} 3$ & & $30(35.3 \%)$ & & \\
\hline $\mathrm{T} 4$ & & $30(35.3 \%)$ & & \\
\hline $\mathrm{pN}^{*}$ & & & $<0.01$ & 0.02 \\
\hline NO & & $26(30.6 \%)$ & & \\
\hline N1 & & $11(12.9 \%)$ & & \\
\hline $\mathrm{N} 2$ & & $20(23.5 \%)$ & & \\
\hline N3 & & $28(12.9 \%)$ & & \\
\hline Positive lymph node ratio* & & & $<0.01$ & 0.03 \\
\hline Extent of lymph node harvest* & & & 0.18 & 0.517 \\
\hline D1 & & $47(55.3 \%)$ & & \\
\hline $\mathrm{D} 1+$ & & $26(30.6 \%)$ & & \\
\hline D2 & & $12(14.1 \%)$ & & \\
\hline $\mathrm{M}^{* *}$ & & & $<0.01$ & 0.22 \\
\hline M0 & & $78(91.8 \%)$ & & \\
\hline M1 & & $7(8.2 \%)$ & & \\
\hline Grade*** & & & $<0.01$ & 0.14 \\
\hline 1 & & $3(4.1 \%)$ & & \\
\hline 2 & & $23(31.1 \%)$ & & \\
\hline 3 & & $48(64.9 \%)$ & & \\
\hline Stage & & & $<0.01$ & 0.02 \\
\hline 0 & & $2(2.3 \%)$ & & \\
\hline I & & $16(18.6 \%)$ & & \\
\hline II & & $19(22.1 \%)$ & & \\
\hline III & & $42(48.8 \%)$ & & \\
\hline IV & & $7(8.1 \%)$ & & \\
\hline Lauren classification $* * * *$ & & & 0.08 & 0.88 \\
\hline Intestinal & & $31(51.7 \%)$ & & \\
\hline Diffuse & & $19(31.7 \%)$ & & \\
\hline Mixed & & $10(16.7 \%)$ & & \\
\hline Resection* & & & $<0.01$ & 0.90 \\
\hline R0 & & $76(89.4 \%)$ & & \\
\hline $\mathrm{R} 1$ & & $4(4.7 \%)$ & & \\
\hline $\mathrm{R} 2$ & & $5(5.9 \%)$ & & \\
\hline
\end{tabular}

Excluding missing patient data: $* \mathrm{n}=1, * * \mathrm{n}=2, * * * \mathrm{n}=12, * * * * \mathrm{n}=26$.

As far as tumor characteristics are concerned, the current study demonstrated that tumor $\mathrm{T}$ - and $\mathrm{N}$-stage, positive lymph node ratio and lymphovascular invasion were significantly associated with positive CD44 expression.
When considering the many roles of CD44 in tumor adhesion, invasion and metastasis, these findings should be expected. All these characteristics are related to the invasive properties of tumor cells. A high expression of CD44 
Table II. CD44 positivity (HSCORE for CD44>30) with patient clinicopathological data, and overall and disease-free survival $(n=83)$.

\begin{tabular}{|c|c|c|}
\hline Characteristic & OR $(95 \% \mathrm{CI})$ & $p$-Value \\
\hline Gender (female) & $1.01(0.83-1.23)$ & 1 \\
\hline Age $>70$ years & $1.04(0.87-1.25)$ & 0.83 \\
\hline pT & & 0.04 \\
\hline $\mathrm{T} 1$ & (reference) & \\
\hline $\mathrm{T} 2$ & $1.51(1.10-2.06)$ & \\
\hline $\mathrm{T} 3$ & $1.44(1.11-1.87)$ & \\
\hline $\mathrm{T} 4$ & $1.38(1.06-1.79)$ & \\
\hline $\mathrm{pN}$ & & 0.04 \\
\hline N0 & (reference) & \\
\hline N1 & $1.31(0.99-1.74)$ & \\
\hline $\mathrm{N} 2$ & $1.17(0.93-1.49)$ & \\
\hline N3 & $1.28(1.02-1.59)$ & \\
\hline Grade & & 0.51 \\
\hline G1 & (reference) & \\
\hline $\mathrm{G} 2$ & $1.27(0.69-2.33)$ & \\
\hline G3 & $1.36(0.75-2.47)$ & \\
\hline Stage & & 0.08 \\
\hline I & (reference) & \\
\hline II & $1.21(0.93-1.58)$ & \\
\hline III & $1.35(1.07-1.70)$ & \\
\hline IV & $1.12(0.79-1.59)$ & \\
\hline Lauren classification & & 0.07 \\
\hline Diffuse & (reference) & \\
\hline Intestinal & $0.77(0.61-0.96)$ & \\
\hline Mixed & $0.82(0.61-1.09)$ & \\
\hline Positive lymph node ratio & $1.46(1.11-1.90)$ & $<0.01$ \\
\hline Lymphovascular invasion & $1.23(1.03-1.46)$ & 0.04 \\
\hline Survival (dead)* & $1.65(0.83-3.28)$ & 0.15 \\
\hline Disease-free survival (relapse)* & $1.18(0.44-3.15)$ & 0.74 \\
\hline
\end{tabular}

Statistically significant results are shown in bold. *Hazard ratio.

contributes to the adhesion of tumor cells to the endothelium, thus promoting lymphvascular invasion, but also enhances post-extravasation events and distant metastasis (1-4).

A recent meta-analysis shed some light on the equivocal findings of the literature. It demonstrated that CD44 was reliable marker in predicting reduced overall and disease-free survival, advanced $\mathrm{N}$-stage and distant metastasis of GC (29). Similarly, CD44 variant 6 (CD44v6) in GC might influence overall survival, while being significantly associated with TNM stage, Lauren type, lymphatic invasion and liver metastasis of the disease. The pooled results showed that CD44v6 was associated with survival in patients with EC. No significant difference in CD44v6 expression was found in patients with different histological types and tumor stages (30). In the same frame, Honing et al. demonstrated that negative expression of CD44 was a significant prognostic factor for disease-free survival of patients with EC (31).

\section{Conclusion}

Our study showed that CD44 antigen expression might be an indicative and descriptive marker of EMT, mainly in gastric cancer patients, reflecting diverse clinicopathological events. However, it stands equivocal on many of the findings of literature indicating a potential prognostic role of CD44 in patients with gastric and esophageal cancer. Thus further prospective studies are mandatory to clarify the gray zones of knowledge.

\section{Conflicts of Interest}

All Authors declare that they have no competing interests.

Informed consent statement: All involved persons (subjects or legally authorized representative) gave their informed consent (written or verbal, as appropriate) prior to study inclusion.

\section{References}

1 Thiery JP and Sleeman JP: Complex networks orchestrate epithelial-mesenchymal transitions. Nat Rev Mol Cell Biol 7(2): 131-142, 2006.

2 Shook D and Keller R: Mechanisms, mechanics and function of epithelial-mesenchymal transitions in early development. Mech Dev 120(11): 1351-1383, 2003.

3 Natalwala A, Spychal R and Tselepis C: Epithelial-mesenchymal transition mediated tumourigenesis in the gastrointestinal tract. World J Gastroenterol 14(24): 3792-3797, 2008.

4 Lee JM, Dedhar S, Kalluri R and Thompson EW: The epithelialmesenchymal transition: new insights in signaling, development, and disease. J Cell Biol 172(7): 973-981, 2006.

5 Huber MA, Kraut N and Beug H: Molecular requirements for epithelial-mesenchymal transition during tumor progression. Curr Opin Cell Biol 17(5): 548-558, 2005.

6 Thiery JP: Epithelial-mesenchymal transitions in tumour progression. Nat Rev Cancer 2(6): 442-454, 2002.

7 Wang T, Ong CW, Shi J, Srivastava S, Yan B, Cheng CL, Yong WP, Chan SL, Yeoh KG, Iacopetta B and Salto-Tellez M: Sequential expression of putative stem cell markers in gastric carcinogenesis. Br J Cancer 105(5): 658-665, 2011.

8 Klonisch T, Wiechec E, Hombach-Klonisch S, Ande SR, Wesselborg S, Schulze-Osthoff K and Los M: Cancer stem cell markers in common cancers - therapeutic implications. Trends Mol Med 14(10): 450-460, 2008.

9 Dhingra S, Feng W, Brown RE, Zhou Z, Khoury T, Zhang R and Tan D: Clinicopathologic significance of putative stem cell markers, CD44 and nestin, in gastric adenocarcinoma. Int J Clin Exp Pathol 4(8): 733-741, 2011.

10 Wakamatsu Y, Sakamoto N, Oo HZ, Naito Y, Uraoka N, Anami $\mathrm{K}$, Sentani K, Oue N and Yasui W: Expression of cancer stem cell markers ALDH1, CD44 and CD133 in primary tumor and lymph node metastasis of gastric cancer. Pathol Int 62(2): 112119, 2012.

11 Li K, Dan Z and Nie Y-Q: Gastric cancer stem cells in gastric carcinogenesis, progression, prevention and treatment. World $\mathrm{J}$ Gastroenterol 20(18): 5420-5426, 2014. 
12 Shi J, Yao D, Liu W, Wang N, Lv H, He N, Shi B, Hou P and Ji $\mathrm{M}$ : Frequent gene amplification predicts poor prognosis in gastric cancer. Int J Mol Sci 13(4): 4714-4726, 2012.

13 Ryu HS, Park DJ, Kim HH, Kim WH and Lee HS: Combination of epithelial-mesenchymal transition and cancer stem cell-like phenotypes has independent prognostic value in gastric cancer. Hum Pathol 43(4): 520-528, 2012.

14 Takaishi S, Okumura T, Tu S, Wang SSW, Shibata W, Vigneshwaran R, Gordon SAK, Shimada Y and Wang TC: Identification of gastric cancer stem cells using the cell surface marker CD44. Stem Cells 27(16): 1006-1020, 2009.

15 Jang BI, Li Y, Graham DY and Cen P: The Role of CD44 in the Pathogenesis, Diagnosis, and Therapy of Gastric Cancer. Gut Liver 5(4): 397-405, 2011

16 AJJC cancer staging manual. (2010). Available at <https:// cancerstaging.org/references-tools/deskreferences/pages/ default.aspx>

17 Yong C-S, Ou Yang C-M, Chou Y-H, Liao C-S, Lee C-W and Lee C-C: CD44/CD24 expression in recurrent gastric cancer: a retrospective analysis. BMC Gastroenterol 12: 95, 2012.

18 Gallatin WM, Weissman IL and Butcher EC: A cell-surface molecule involved in organ-specific homing of lymphocytes. Nature 304(5921): 30-34, 1983.

19 Idzerda RL, Carter WG, Nottenburg C, Wayner EA, Gallatin WM and St John T: Isolation and DNA sequence of a cDNA clone encoding a lymphocyte adhesion receptor for high endothelium. Proc Natl Acad Sci USA 86(15): 4659-4663, 1989.

20 Takahashi E, Nagano O, Ishimoto T, Yae T, Suzuki Y, Shinoda T, Nakamura S, Niwa S, Ikeda S, Koga H, Tanihara H and Saya $\mathrm{H}$ : Tumor necrosis factor-alpha regulates transforming growth factor-beta-dependent epithelial-mesenchymal transition by promoting hyaluronan-CD44-moesin interaction. J Biol Chem 285(6): 4060-4073, 2010.

21 Ueyama H, Yao T, Nakashima Y, Hirakawa K, Oshiro Y, Hirahashi M, Iwashita $A$ and Watanabe $S$ : Gastric adenocarcinoma of fundic gland type (chief cell predominant type): proposal for a new entity of gastric adenocarcinoma. Am J Surg Pathol 34(5): 609-619, 2010.

22 Xu G, Zhang W, Sun Q, Xu X, Zou X and Guan W: Combined epithelial-mesenchymal transition with cancer stem cell-like marker as predictors of recurrence after radical resection for gastric cancer. World J Surg Oncol 12: 368, 2014.

23 Zöller M: CD44: can a cancer-initiating cell profit from an abundantly expressed molecule? Nat Rev Cancer 11(4): 254-267 2011 .
24 Doventas A, Bilici A, Demirell F, Ersoy G, Turna H and Doventas Y: Prognostic significance of CD44 and c-erb-B2 protein overexpression in patients with gastric cancer. Hepatogastroenterology 59(119): 2196-2201, 2012.

25 Ghaffarzadehgan K, Jafarzadeh M, Raziee HR, Sima HR, Esmaili-Shandiz E, Hosseinnezhad H, Taghizadeh-Kermani A, Moaven $\mathrm{O}$ and Bahrani M: Expression of cell adhesion molecule CD44 in gastric adenocarcinoma and its prognostic importance. World J Gastroenterol 14(41): 6376-6381, 2008.

26 Sheridan C, Kishimoto H, Fuchs RK, Mehrotra S, BhatNakshatri P, Turner $\mathrm{CH}$, Goulet R, Badve S and Nakshatri H: CD $44^{+} / \mathrm{CD} 24^{-}$breast cancer cells exhibit enhanced invasive properties: an early step necessary for metastasis. Breast Cancer Res 8(5): R59, 2006.

27 Tsukita S, Oishi K, Sato N, Sagara J, Kawai A and Tsukita S: ERM family members as molecular linkers between the cell surface glycoprotein CD44 and actin-based cytoskeletons. J Cell Biol 126(2): 391-401, 1994.

28 Jung WY, Kang Y, Lee H, Mok Y-J, Kim HK, Kim A and Kim B-H: Expression of moesin and CD44 is associated with poor prognosis in gastric adenocarcinoma. Histopathology 63(4): 474481, 2013.

29 Sanaat Z, Halimi M, Ghojezadeh M, Pirovi AH, Gharamaleki JV, Ziae AEJE and Kermani IA: Immunohistochemical analysis of p53, Ki-67, CD44, HER-2/neu expression patterns in gastric cancer, and their association with one year survival in north-west of Iran. Int J Hematol Oncol Stem Cell Res 7(3): 15-20, 2013.

$30 \mathrm{Wu}$ Y, Li Z, Zhang C, Yu K, Teng Z, Zheng G, Wang S, Liu Y, Cui L and Yu X: CD44 family proteins in gastric cancer: a metaanalysis and narrative review. Int J Clin Exp Med 8: 3595-3606, 2015.

31 Honing J, Pavlov KV, Meijer C, Smit JK, Boersma-van Ek W, Karrenbeld A, Burgerhof JGM, Kruyt FAE and Plukker JTM: Loss of CD44 and SOX2 expression is correlated with a poor prognosis in esophageal adenocarcinoma patients. Ann Surg Oncol 21(Suppl 4): S657-664, 2014.
Received September 23, 2016

Revised October 8, 2016

Accepted October 12, 2016 